\title{
Influence and Risk Assessment of Climate Change in Airport of China based on Hesitant Analytic Hierarchy Process and TOPSIS
}

Chenyang Song ${ }^{1}$, Ren Zhang ${ }^{1}$, Taiping Hou ${ }^{2}$, Chengzu Bai ${ }^{1}$, Kefeng Liu ${ }^{1}$, Qiang Lon ${ }^{2}$ 1College of Meteorology and Oceanography, PLA University of Science and Technology, Nanjing 211101,

China

2Meteorological bureau in Tangshan Caofeidian Industrial Zone in Hebei province, Tangshan 063000,China

\section{基于犹豫层次分析-TOPSIS 的空军机场气候变化 风险评估}

\begin{abstract}
Based on complexity and uncertainty of natural environmental changes in airport in the background of global climate change, in view of increasing momentum of marine meteorological disaster, we explored the risks of climate change in six major airports in China's coastal areas. Confronting with sophisticated social and natural factors for effective evaluation, this paper put forward a new method which combines Hesitant Analytic Hierarchy Process and TOPSIS, and designed a climate change risk assessment model for airport in order to provide recommendations for port construction and policies in face of climate change. Moreover, the methods and approaches of analysis will supply technical references to other climatic disaster assessment and risk prevention.
\end{abstract}

Keywords: climate change; risk assessment; airport; Hesitant Analytic Hierarchy Process; TOPSIS

\section{摘要}

针对气候变化背景下空军机场可能面临的威胁挑战, 从风险分析角度提出了空军机场气候变化风险概念 模型, 为解决风险评估建模时决策者难以有效评判的 情况，构建了犹豫层次分析法和逼近于理想解的排序 沿海地区 6 个空军机场作为仿真算例进行了分析验 证。结果表明, 所建模型能够合理构建空军机场气候 变化风险评估指标体系, 得到与事实接近的量化评估 结果, 研究思想和方法途径可为其他气候灾害评估和 风险防范提供参考。

关键词: 空军机场; 气候变化; 犹豫层次分析法; TOPSIS; 风险评估

\section{引言}

根据 IPCC 第五次评估报告, 1880-2012 年期间, 全球平均地表温度升高了 $0.85^{\circ} \mathrm{C}[1]$; 采用全球比较 计划第五阶段（CMIP5）耦合模式, 预估未来全球气 候变暖仍将继续[2]。气候系统的变化会长期显著地 影响军事活动和目标区域的天气条件, 从而制约军事 活动的形态。Sweeny ${ }^{[3]}$ 发现 ENSO 事件带来的大雾、 降雨、降雪和仪器起飞条件等的频率变化, La Joie ${ }^{[4]}$ 认为降水量变量是对非洲之角所有军事行动影响最 大的因子。气候系统的变化会反馈到自然地理环境和 社会经济系统, 造成战场环境形态的改变, 从而影响 到军事任务和战略布局的调整。Jack Huntley ${ }^{[5]}$ 研究 了气候变化及其对美国工兵部队要做好供水系统调 整、海岸堤防加固等任务准备以应对气候变化可能带 来的水资源分布不均匀、海平面上升以及不断增加的 
Risk Analysis and Crisis Response in Big Data Era (RAC-16)

风且风、风暴潮等危害。Cheney ${ }^{[6]}$ 通过损失成本模型, 指出美军的 Diego Garcia 等五个军事基地面临着最 大的气候变化及海平面上升风险。我军亦提出气候变 化与国家海洋战略的影响与风险评估 ${ }^{[7]}$, 彭鹏 ${ }^{[8]}$ 对气 候变化与战场环境影响进行了相关研究。虽然上述研 究工作对气候变化影响有了较明确的认识, 但基本上 还偏向于定性的研讨, 对气候变化相关的定量化综合 风险评估研并不多。

量化的风险评估是风险管理的主流和核心环节 ${ }^{[9]}$ 。现实决策问题中, 由于决策问题的复杂性以及决 策者自身的知识和经验不足等因素, 致使决策者常常 难以判别决断。朱斌基于传统的层次分析法 (Analytic Hierarchy Process, AHP), 引入犹豫偏 好的概念, 用概率分布描述犹豫偏好, 并将其运用到 层次分析法中, 提出犹豫层次分析法 (H-AHP) ${ }^{[10]}$ 。 它适用于决策者做判断时犹豫不决的情况, 可描述决 策者提供的有多个可能值的偏好信息, 且这些可能值 不需要集成或修改, 提高了准确度和决策者对最终方 案排序结果的满意程度。TOPSIS 是一种逼近理想解 的排序法, 是多目标决策分析中常用的有效方法。它 通过计算某一方案与正理想解、负理想解之间的加权 欧式距离, 得出该方案与正理想解的接近程度, 以此 作为评价各方案优劣的依据。

本文中空军机场气候变化风险是指气候变化背 景下空军机场可能遭受破坏的可能性以及由其造成 损失的严重程度。由于空军机场气候变化风险评估是 一个涉及气候、生态、自然环境、防护工程、灾害预 报预警能力及应急响应机制等的多方面、多目标、多 层次决策问题, 需综合考虑不同评估方法和模型的优 缺点。为此, 本文引入 H-AHP和TOPSIS相结合的研究 思想和方法 ${ }^{[11]}$, 进行所选空军机场之间的风险评估。 该研究的核心是利用基于犹豫层次分析法 (H-AHP), 建立空军机场气候变化风险评估指标体系, 计算各指 标的权重; 然后将指标权重和各评估单元的无量纲化 决策矩阵相结合, 形成加权决策矩阵; 利用TOPSIS 法计算出加权决策矩阵的正理想解、负理想解、各评 估单元与正理想解的欧式距离、贴近度, 据此来确定 气候变化背景下各空军机场风险的大小。各评估空军 机场与正理想解之间的贴近度越大, 其气候变化风险 越高。数据资料和研究方法

\section{1 风险评价指标体系}

本文在空军机场气候变化风险要素识别的基础 上, 首先构建气候变化风险评价指标体系, 随后引入 犹豫层次分析方法和 TOPSIS 法, 研究建立我国空军 机场气候变化风险评估模型, 并以我国六个主要空军
机场为例进行仿真模拟。

根据空军机场气候变化风险成因、自然因素与管 理背景以及主要风险要素的分析, 将空军机场变化风 险因素大致分为 3 个方面: 气候变化危险性、机场地 理与功能的脆弱性以及风险防范能力。

气候变化的危险性主要包括: 能见度、低云、大 风、暴雨和降水性天气等自然因素。一般认为, 低能 见度日越多, 能见度越差, 越不利于日常工作和作战 训练, 其危险性越大。低云直接影响飞机的起降。 平均风速越大, 越不利于正常训练, 则风险危险越大; 定常风向变化越大, 越不利于正常训练。对流性降水 量越大, 发生雷暴天气的概率越大; 对流性降水量越 小, 发生雷暴天气的概率和强度越小。降水量越稳定, 生态变迁的可能性越小, 降水影响越小。

机场地理与功能脆弱性主要反映机场地理特征、 机场规模和机场主要装备等的脆弱性。机场的地理特 征指机场的地理位置、地形地貌及周边生态环境。一 般认为, 机场距离城市越远, 地形越复杂, 周边生态 越复杂, 受气候变化影响越敏感。机场规模脆弱性是 对机场建设防护等级、配套设施水平的综合评价, 评 价依据是机场吞吐能力。装备脆弱性指数是对场站主 要装备的技术水平、装备强度的评价, 评价依据是平 均起飞重量和平均服役年限。

风险防范能力一般针对机场防护工程设施、灾害 预报警报能力、应急响应机制与预案等几个方面进行 评价。机场防护工程设施包括机场的建设使用年限、 基础设施、设计标准、建设质量及技术水平等; 灾害 预报警报能力指针对强风、暴雨、恶劣能见度等极端 天气的预报、警报技术和能力水平; 应急响应机制与 预案指突发极端天气和气象灾害的应急响应机制和 联动措施、处置能力、物质保障及救援行动协调水平 等。

综上分析, 建立空军机场气候变化风险评价体 系, 如表 1 所示。

\section{2.风险因素权重的客观确定}

风险分析和风险评估建模中, 层次分析法是一个定性 与定量结合的便捷方便的系统分析方法。现实决策问 题中, 由于决策问题的复杂性以及决策者自身知识和 经验不足等因素, 致使决策过程不仅存在主观性、人 为性, 还存在决策者面对两个类似或相邻的选项难以 判别的情况。为此, 朱斌基于传统的层次分析法, 引 入犹豫偏好的概念, 用概率分布描述犹豫偏好, 并将 其运用到层次分析法之中, 提出了犹豫层次分析法 (H-AHP)。H-AHP 适用于决策过程中决策者选择、判 
Risk Analysis and Crisis Response in Big Data Era (RAC-16)

\section{表 1 空军机场气候变化风险评价体系结构}

Table.1 Risk assessment structure of climate change in Air force Airport

\begin{tabular}{|c|c|c|}
\hline \multirow{11}{*}{ 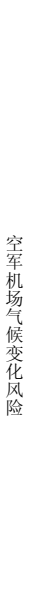 } & \multirow{5}{*}{ 危险性 H } & 能见度影响指数 C1 \\
\hline & & 低云影响指数 C2 \\
\hline & & 大风影响指数 C3 \\
\hline & & 暴雨影响指数 C4 \\
\hline & & 降水影响指数 C5 \\
\hline & \multirow{3}{*}{ 脆弱性 V } & 地理特征指数 C6 \\
\hline & & 规模脆弱性指数 C7 \\
\hline & & 装备脆弱性指数 C8 \\
\hline & \multirow{3}{*}{ 防范能力 $\mathrm{R}$} & 防护功能设施 C9 \\
\hline & & 灾害预报警报能力 $\mathrm{C} 10$ \\
\hline & & 应急响应机制与预案 $\mathrm{C} 11$ \\
\hline
\end{tabular}

断时犹豫不决的情况, 可描述决策者提供的有多个可 能值的偏好信息, 且这些可能值不需要集成或修改, 进而提高了准确度和决策者对最终方案排序结果的 满意程度。

针对气候变化的影响评估特性, 引入犹豫层次分 析法, 通过各指标间相对重要程度的评价建立概率型 犹豫积型偏好关系, 并应用蒙特卡洛模拟方法, 计算 同一层次相应元素对于上一层次某元素相对重要性 的排序权值。再从结构底层开始, 对于上一层次中元 素, 集成方案的排序权值, 直到获得方案对于控制属 性的综合排序权值，从而客观、定量地获取各相关指 标的权重 ${ }^{[12]}$ 。

\section{1 构造概率型犹豫积型偏好关系}

对于一个控制属性, 将决策问题分解, 构造从上到下 的结构层次, 包括控制层、属性层和方案层。每一层 次还可包含子层次。可将空军机场气候变化风险分为 气候变化危险性 B1、机场脆弱性 B2 和风险防范能力 B3, 各层次结构如表 1 所示。对一个集合 $X=\left\{x_{1}, x_{2}, \cdots, x_{n}\right\}$, 假设决策者对 $X$ 中元素进行两 两比较, 然后给出概率型犹豫偏好信息, 根据专家意 见构造概率型犹豫积型偏好关系 ( P-HMPR) $Y=\left(y_{i j}\right)_{n \times n}$, 其中 $y_{i j}=\left(y_{i j}^{(l)}\left(p_{i j}^{(l)}\right)|l=1, \cdots,| y_{i j} \mid\right)$, $|y|$ 是 $y$ 中可能值的数量, $y_{i j}$ 表示 $x_{i}$ 对 $x_{j}$ 的偏好度, 且满足

$y_{i j}^{\rho(l)} y_{j i}^{\rho(l)}=1, \quad y_{i i}=1, \quad\left|y_{i j}\right|=\left|y_{j i}\right|, \quad p_{i j}^{\rho(l)}=p_{j i}^{\rho(l)}$, $i, j=1,2, \cdots, n$

$y_{i j}^{\rho(l)} \leq y_{i j}^{\rho(l+1)}, \quad i<j$ 。

式中: $y_{i j}^{\rho(l)}$ 是 $y_{i j}$ 的第 $\rho$ 个可能值, $p_{i j}^{\rho(l)}$ 是 $y_{i j}^{\rho(l)}$ 的 概率。

根据我国空军机场气候变化风险评估指标体系, 基于 1-9 标度, 邀请 5 名相关专家以属性层中的每一 个属性为支配元素, 进行两两比较, 给出犹豫偏好信 息, 并依据评估结果, 构造概率型犹豫积型偏好关系 (P-HMPR)。

\section{2 对 P-HMPR 进行一致性检验}

基于概率分布 $p_{i j}$, 从 $y_{i j}$ 中随机选择 $y_{i j}^{(l)}$, 可得一个 MPR $Y^{(l)}=\left(y_{i j}^{(l)}\right)_{n \times n} \circ Y^{(l)}$ 的几何一致性指标, 表示为 $G C I{ }_{Y}(l)$ 。根据 Crawford 和Williams ${ }^{[13]}$ 提出 的行几何平均法 (RGMM), 对于 
Risk Analysis and Crisis Response in Big Data Era (RAC-16)

$$
\operatorname{MPR}_{Y}(l)=\left(y_{i j}^{(l)}\right)_{n \times n}
$$

方案 $x_{i}$ 的排序值 $\omega_{i}$ 为 $Y^{(l)}$ 中行元素的几何平均 数:

$$
\omega_{i}=\frac{\left(\Pi_{j=1}^{n} y_{i j}^{(l)}\right)^{\frac{1}{n}}}{\sum_{i=1}^{n}\left(\Pi_{j=1}^{n} a_{i j}\right)^{\frac{1}{n}}}
$$

基于排序值 $\omega_{i}$, Aguaron ${ }^{[14]}$ 等提出了几何一致性 指标 $(\mathrm{GCI})$ 用于检验 $Y^{(l)}$ 的一致性水平:

$$
G C I_{Y^{(l)}}=\frac{2}{(n-1)(n-2)} \sum_{i<j} \log ^{2} e_{i j} 。
$$

式中: $e_{i j}=y_{i j}^{(l)} \omega_{j} / \omega_{i}$ 。

令 $Y$ 为一个犹豫偏好空间, 那么 $Y$ 的期望集合 一致性指标可以定义为

$$
E(G C I)_{Y}=\left(\prod_{i, j=1}^{n} \frac{1}{\left|y_{i j}\right|}\right) \sum_{Y} G C I Y_{Y}(l)
$$

$E(G C I)_{Y}$ 的计算基于蒙特卡洛模拟。

\section{3 计算各指标权重}

基于行几何平均法 (RGMM), 应用蒙特卡洛模拟方 法,计算同一层次相应元素对于上一层次某元素相对 重要性的排序权值。再从结构的底层开始, 对于上一 层次中元素, 集成方案的排序权值, 直到获得方案对 于控制属性的综合排序权值, 即各相关指标的权重 ${ }^{[16]}$, 结果如表 7 所示, 在一级指标中, 气候变化危险 性所占权重最大, 机场脆弱性次之, 保障防范能力最 小, 评估结果比较符合实际情况。

\section{3 用 TOPSIS 方法评估建模}

在确定各指标权重的基础上，应用 TOPSIS 法计算气 候变化背景下空军机场风险。TOPSIS 法的核心思想 是通过计算某一方案与正理想解、负理想解之间的加 权欧式距离, 得出该方案与正理想解的接近程度, 以 此作为评价各方案的依据。

\section{1 构造决策矩阵}

设参与评价的多指标决策问题的方案集为

$$
M=\left(M_{1}, M_{2}, \cdots, M_{m}\right),
$$

指标集为 $C=\left(C_{1}, C_{2}, \cdots C_{n}\right)$, 方案 $M_{i}$ 对指标 $C_{j}$ 的 值记为 $x_{i j}(i=12, \cdots, m ; j=1,2, \cdots, n)$, 则决策矩 阵为:

$$
X=\left[\begin{array}{ccccc} 
& C_{1} & C_{2} & \cdots & C_{n} \\
M_{1} & x_{11} & x_{12} & \cdots & x_{1 n} \\
M_{2} & x_{21} & x_{22} & \cdots & x_{2 n} \\
\vdots & \vdots & \vdots & \vdots & \vdots \\
M_{m} & x_{m 1} & x_{m 2} & \cdots & x_{m n}
\end{array}\right]
$$

本文选取东南地区 6 个空军机场作为实证研究 对象, 根据所取得的数据, 通过问卷调查, 请相关领 域的专家按照 0-1 打分法, 对空军机场的环境要素、 物理要素及防范能力等进行评价打分。

本文中 $M_{1}, M_{2}, \ldots M_{6}$ 依次为选取的 6 个空军 机场。根据专家打分情况构建决策矩阵 $X$ 。

\section{2 决策矩阵标准化}

为了消除各指标量纲不同对方案决策带来的影响, 需 要对决策矩阵进行无量纲化处理, 构建标准化矩阵 $V=\left(v_{i j}\right)_{n \times n}$ 。

对于值越大风险越大型指标:

$$
v_{i j}=\frac{x_{i j}-\min \left(x_{j}\right)}{\max \left(x_{j}\right)-\min \left(x_{j}\right)}
$$

对于值越小风险越大型指标:

$$
v_{i j}=\frac{\max \left(x_{j}\right)-x_{i j}}{\max \left(x_{j}\right)-\min \left(x_{j}\right)} 。
$$

其中 $v_{i j}$ 为 $x_{i j}$ 归一化后的值, $\max \left(x_{j}\right) 、 \min \left(x_{j}\right)$ 分

别为第 $j$ 个指标的最大值和最小值。

对决策矩阵 $X$ 无量纲化处理, 得到标准化矩阵 V。

\section{3 构建加权标准化决策矩阵}

将用 H-AHP 求得的指标权重 $W$ 与无量纲化矩阵 
Risk Analysis and Crisis Response in Big Data Era (RAC-16)

$V$ 相乘, 得到加权标准化决策矩阵 $R=\left(r_{i j}\right)_{m \times n}$, 其中 $r_{i j}=\omega_{j} \bullet v_{i j}, \omega_{j}$ 为各指标的权重值。

\section{4 计算正理想解和负理想解}

分别确定每个指标集的最大值与最小值, 并以正 向指标的最大值与负向指标的最小值构成正理想解, 以正向指标的最小值与负向指标的最大值构成负理 想解。

计算公式如下:

$$
S_{j}^{+}=\max _{1 \leq i \leq m}\left\{r_{i j}\right\},
$$

$C_{j}$ 为值越大风险越大型指标;

$\min _{1 \leq i \leq m}\left\{r_{i j}\right\}, C_{j}$ 为值越小风险越大型指标；

$S_{j}^{-}=\min _{1 \leq i \leq m}\left\{r_{i j}\right\}, C_{j}$ 为值越大风险越大型指

标;

$$
\max _{1 \leq i \leq m}\left\{r_{i j}\right\}, C_{j} \text { 为值越小风险越大型指标。 }
$$

\section{5 计算样本与正理想解和负理想解间的欧式距离}

设样本 $i$ 到正理想解和负理想解之间的欧式距离

分别为 $S d_{i}^{+}$和 $S d_{i}^{-}$, 则可得到:

$$
S d_{i}^{+}=\sqrt{\sum_{j=1}^{n}\left(S_{j}^{+}-r_{i j}\right)^{2}}
$$

$S d_{i}^{-}=\sqrt{\sum_{j=1}^{n}\left(S_{j}^{-}-r_{i j}\right)^{2}}$ 。式中: $i=1,2, \cdots m$ 。

根据正负理想解, 计算样本到正、负理想解的欧式 距离。

\section{6 计算相对贴近度及结果分析}

计算相对贴近度, 实现样本的排序优选。各样本与 正理想解的相对贴近度为 $\eta_{i}$,

$$
\eta_{i}=\frac{S d_{i}^{-}}{S d_{i}^{+}+S d_{i}^{-}}, \quad i=1,2, \cdots m
$$

反映了待评价样本与正理想解在态势变化上的 接近程度, 根据 $\eta_{i}$ 的大小对样本进行排序, $\eta_{i}$ 越大, 表示待评价样本越贴近正理想解, 样本风险越大。根 据样本到正、负理想解的欧式距离, 采用上式计算样 本的相对贴近度。一般评价中, 越贴近正理想解的方 案是越好的, 但是由于本文将犹豫层次分析法和 TOPSIS 法相结合应用到气候变化背景下空军机场的 风险评估中, 风险评估的结果是越小越好, 所选空军 机场越贴近正理想解说明其可能面临的气候变化灾 害风险越大。因此, 由加权标准化决策矩阵 $R$ 和相 对贴近度 $\eta_{i}$ 的计算结果可知, 机场 $\mathrm{M} 2$ 得分最低, 说 明其风险最小, 机场 M5 得分最高, 说明其风险最大。

\section{4 结束语}

传统的 TOPSIS 法常常需要人为确定各方案不同 属性的权重, 具有较大的主观性和不确定性。本文基 于气候变化背景下我国可能面临的风险分析, 通过犹 豫层次分析法和 TOPSIS 法相结合的方法, 建立了气 候变化背景下空军机场威胁风险评价体系和风险评 估模型, 利用犹豫层次分析法客观、定量地确定了各 属性权重, 提高了准确度和决策者对最终方案排序结 果的满意程度。针对我国空军机场气候变化风险进行 评估建模和仿真实验, 实现了空军机场气候变化风险 的量化评估。分析结果可为空军机场应对气候变化风 险提供客观定量的决策参考, 评估方法和建模过程也 可为其他缺乏精确数据的风险研究提供思路。

鉴于气候变化背景下空军机场风险的影响因子 众多、机理过程复杂, 本文仅在有限层面上进行了优 化处理, 方法模型效果有待进一步论证和实践检验。 此外, 由于气候变化预测的不确定性, 机场相关可获 取资料有限, 构建的空军机场威胁风险指标还比较简 单, 指标的量化有待完善。下一步工作重点是对指标 进一步充实, 探索更有效的量化评估方法, 以使评估 结果更加客观、准确。

\section{致谢}

本研究得到了国家自然科学基金项目 (41276088) 和 山市曹妃甸工业区专项（CQZ-2014001）的资助。

\section{参考文献}

[1] IPCC. Climate change 2013:the physical science basis.http://www.ipcc.ch/report/ar5/wg1/\#.Uq-tD7 KBRR1. 
Risk Analysis and Crisis Response in Big Data Era (RAC-16)

[2] IPCC. Summary for policymakes.

http://www.ipcc.ch/pdf/assessment-report/ar5/wg1 /WG1AR5-SPM-FINAL.pdf.

[3] John Neander. El Nino Its Far-Reaching Environmental Effects on Army Tactical Decision Aids. AD-A307126,1996.

[4] Jack E.Huntley and John E.Neander. Global climate change(GCC) issues and their impacts on the US army corps of engineers. AD-A247279, 1991.

[5] Stephen A. Cheney, Nick Cunningham. Strategic bases vulnerable to climate change

[6] CAN. National Security and the Threat of Climate Change.AD-A469156,2007.

[7] 李倩.全球气候变化对国家海洋战略的影响评估 与风险分析.南京:解放军理工大学,2011.

[8] 彭鹏.气候变化与战场环境影响关联及风险评估 研究.南京:解放军理工大学,2011.

[9] 张继权,李宁.主要气象灾害风险评价与管理的数 量化方法及其应用.北京: 北京师范大学出版 社, 2007 .

[10] 朱斌.基于偏好关系决策方法及应用研究.南京:
东南大学, 2014.

[11] 何逢标.塔里木河流域水权配置研究.南京:河海 大学, 2007.

[12] 宋晨阳, 王锋, 张韧, 等.气候变化背景下我国 城市高温热浪的风险分析与评估.灾害学, 2016,31 (4) : 201-206.

[13] Crawford G, Williams C.A note on the analysis of subjective judgment matrices. Journal of Mathematical Psychology .1985, 29(4):387-405.

[14] Aguaron J,Moreno-Jimenez J Ma. The geometric consistency index : Approximated thresholds. European Journal of Operational Research. 2003, 147(1):137-145.

[15] Ren Zhang, Hengwan Zou, Mei Hong. Risk Analysis of Water Resources Crisis in the Lancang - Mekong River Drainage Basin under the Background of Climate Change. Journal of Risk Analysis and Crisis Response. 2012, 2-3: 209-213.

[16] Dengfeng Liu,Dong Wang,Yuankun Wang. Eutrophication Hazard Evaluation Using Copula-Cloud. Journal of Risk Analysis and Crisis Response. 2016,6-1:10-14. 